Research Article

\title{
Quality-Oriented Classification of Aircraft Material Based on SVM
}

\author{
Hongxia Cai, Tingting Yu, and Chenglong Xia \\ Shanghai Key Laboratory of Intelligent Manufacturing and Robotics, School of Mechatronic Engineering and Automation, \\ Shanghai University, Mailbox 232, No. 149 Yanchang Road, Shanghai 200072, China \\ Correspondence should be addressed to Hongxia Cai; donnayu499@gmail.com
}

Received 17 June 2014; Revised 13 September 2014; Accepted 14 September 2014; Published 9 November 2014

Academic Editor: Yan-Wu Wang

Copyright (C) 2014 Hongxia Cai et al. This is an open access article distributed under the Creative Commons Attribution License, which permits unrestricted use, distribution, and reproduction in any medium, provided the original work is properly cited.

\begin{abstract}
The existing material classification is proposed to improve the inventory management. However, different materials have the different quality-related attributes, especially in the aircraft industry. In order to reduce the cost without sacrificing the quality, we propose a quality-oriented material classification system considering the material quality character, Quality cost, and Quality influence. Analytic Hierarchy Process helps to make feature selection and classification decision. We use the improved Kraljic Portfolio Matrix to establish the three-dimensional classification model. The aircraft materials can be divided into eight types, including general type, key type, risk type, and leveraged type. Aiming to improve the classification accuracy of various materials, the algorithm of Support Vector Machine is introduced. Finally, we compare the SVM and BP neural network in the application. The results prove that the SVM algorithm is more efficient and accurate and the quality-oriented material classification is valuable.
\end{abstract}

\section{Introduction}

There is no doubt that aircraft industry should pay more attention to quality. For the purpose of ensuring the quality of aircraft, aircraft factories have made a lot of study and most of them have established quality management system. However, there are so many parts in one airplane, and even if one screw has quality problems, it may cause catastrophic damage. As a result, the aircraft factories are facing greater challenges in the quality control and management. The quality of aircraft materials determines the quality of the aircraft directly. Therefore, the quality of aircraft material should be guaranteed. However, it is almost impossible to do continuous quality inspection of each aircraft material for there are large numbers of component parts of an aircraft, more than millions [1]. As the materials have different quality-related character, we believe it is more feasible to take different strategies for the different materials in the management of quality. Obviously, some materials are common while others are rare. Some materials are easy to meet the quality standards while others are not. It is inevitable that we should pay more attention to some of the materials, but we may pay little attention to the other materials.
Therefore the classification considering quality characters will improve the quality and the reliability in the aircraft industry. However, there is no research on the material classification considering the quality-related attributes. In all fields, the material classification in general is mainly oriented to inventory management based on $\mathrm{ABC}$ inventory control, usually considering material quantity, purchase cost, and so on. In the aircraft industry, the materials are so extensive and complex that it is difficult to realize the classification for the inventory control, let alone the management of the quality.

For material classification, $\mathrm{ABC}$ analysis originated by Dickie [2] based upon the Pareto principle is one of the most widely used tools. Nevertheless, ABC inventory control is not appropriate to use for precise classification problems with multicriteria. Among the contributions to multicriteria inventory classification (MCIC), there are Analytic Hierarchy Process classification method, Kraljic Portfolio Matrix, and so on. AHP is an effective way for feature selection in classification [3]. Analytic Hierarchy Process (AHP) was first developed for decision making by Saaty [4] and extended by Moran et al. [5] who have developed a more specific method directly for design decision making. Pedrycz and Song [6] applied AHP for group decision making. AHP is 
also applied to determine the important weights of customer requirements in quality function deployment. Kwong and Bai [7] used the extent analysis method and the principles for the comparison of fuzzy numbers to determine the important weights for the customer requirements in QFD. Meanwhile, traditional Kraljic Portfolio Matrix was firstly applied to divide the goods into strategic items, leveraged items, Bottleneck-type items, and general items depending on the two criteria of material supply: profit and risk by using a second-order matrix. After that, many scholars make different application and improvement to this method. Lee and Drake [8] verified the significance of the classification model for manufacturing enterprises. Terpend et al. [9] pointed out that material plans should combine with Kraljic matrix after demonstrating material classification model by clustering method.

In the application of the algorithm for classification, some scholars are interested in the artificial intelligence and machine learning such as genetic algorithm (GA) [10], artificial neural network (ANN) [11], k-nearest neighbors (kNN) [12], and particle swarm optimization method (PSO) [13]. Most machine learning methods need sufficient and high quality sample data [14]. Besides, some of the algorithms are "dimension disaster" and "too much learning." However, there is not enough history data for the limit of our country's aviation industry and aircraft material classification problem belongs to typical nonlinear issue. It leads to the fact that the performance of the model for most of the classification algorithms will degrade. Support Vector Machine (SVM) developed by Vapnik is based on the statistical learning and minimum structural risk theory. SVM is fast becoming one of the most used methods for prediction classification [15]. SVM owns few adjustable parameters, quick computing speed, accurate classification rate, good robustness, and good generalization ability [16]. It solves the nonlinear, high dimension, and local minima and some other issues effectively. In the absence of more background information given, it can also achieve high prediction accuracy $[17,18]$. SVM is applied to mine data and analyze the thermal performance of coolpipes in [19]. Zhu and Blumberg [20] used SVM algorithm for classification processing of ASTER data; they pointed that the classification results show the approach based on SVM has high performance in convergence, speed, and accuracy of training and classifying. SVM is used in the classification of academic texts [21]. Hernández et al. apply SVM to deal with the classification problem of failure identification in the distribution system of electric power [22]. Some scholars also use SVM in signal classification [23]. SVM is also combined with some other algorithms in some researches. For example, Yu et al. [24] successfully applied optimized PSO-SVM to classify the fault type of rolling bearing. Wei [25] used GA (genetic algorithm) and PCA (principal component analysis) combined with improved SVM to complete realtime vehicle classification. The classification precision was improved greatly in his paper and it achieved a high accuracy. Ma et al. [26] proposed a multiple classifier method of binary tree based on SVM. Based on the contribution of so many scholars, we believe SVM is feasible in classification of aircraft material.
Of course, aircraft companies take the material management seriously. For example, Boeing has invested nearly 2 billion to realize the management of production resources. In Airbus Fasteners' Supply Chain Optimization Plan, they simplify the logistics by determining the material qualitatively and quantitatively. China also makes a lot of effort to material management during the project of ARJ21 [27]. However the researches on the material management pay more attention to the supply management [28, 29]. There are also some related studies which mainly focus on spare parts inventory [30-32]. About the research on classification, it is introduced that the material of the aircraft is normally divided into raw material, airborne system finished products, airborne structural components, self-made parts, and so on in [33]. Wu et al. [34] applied SVM in their study for aircraft material classification in which the material is divided into normal material, cost-first material, supply-first material, and so on. Mancini et al. [35] divided the material into TATS (time and temperature sensitive material) and auxiliary for the using of RFID (radio frequency identification). From these papers, we can see that the result of material classification is different for the different purpose. For the quality control in the aircraft industry, Davies et al. [36] present the survey of fatigue failures in helicopter components and discussed the importance of the material quality control for aircraft industry. Shalin and Kantsevich [37] also emphasized that the quality of aircraft materials was a guarantee of aircraft quality and reliability. Hillermeier et al. [38] proposed that quality assurance testing of prepreg materials in the commercial airplane environment was very complex and costly so we should study the material properties to reduce and standardize testing. Therefore, the classification considering quality characters is significant. In this paper, we establish a new quality-oriented material classification system to improve the quality control and management in the aircraft industry. We apply AHP for feature selection and classification decision because the aircraft materials have multiple quality-related attributes in Section 2. In Section 3, we improve the Kraljic method and establish a three-dimensional quality-oriented classification model in accordance with the attributes decided by AHP. In Section 4, the SVM is introduced because it is the best algorithm for small sample classification and regression problems. When we use the SVM algorithm, we maximize classification interval optimal hyperplane to improve the generalization ability of learning machine. Through the study of excellent training samples, it is more accurate to classify unknown types of materials. Its performance is evaluated with the comparison of BP neural network. Finally, in Section 5, we discussed the contribution and limitations of the study.

\section{Quality-Oriented Evaluation System of Civil Aircraft Materials}

2.1. Analysis of Traditional Classification Method. A general aircraft contains more than millions of parts. The aircraft materials also cover a very wide range including raw materials 
purchased by businesses, homemade semifinished products, outsourcing semifinished products, finished products, office supplies, tools and even drawings, and documents. The role of quality played in the classification is ignored, so the traditional unified classification system exposes the following shortcomings.

(1) Difficult to Control the Cost of Quality. The aircraft is assembled by millions of parts. The poor-quality materials indeed affect the quality of aircraft. The aircraft can operate well only if all materials are well qualified. However, some materials are common while others are rare. Some materials are easy to meet the quality standards while others are not. Therefore it is inevitable that some of the materials need enough focus, while others only require relatively less focus. Only if we pay different attention to the different materials, it is possible to reduce the cost without scarifying the quality.

(2) Difficult to Solve the Quality Problems in Time. If there is a quality problem with one material, it will influence the entire production schedule. However, the depth and scope of the influence depend on the quality character of the material. Some materials are designed for a specific type of aircraft. Besides, once there are some quality problems with the complex parts, they need a long time to repair. In addition, some parts affect a wide range of the total quality and some parts are being monopolized by some suppliers. Once these materials appeared to have some quality problems, they will expose a serious influence at a large extent. Thus, we should pay more attention to these materials for early prevention.

Through the above analysis, it is necessary to further propose another classification system according to several indicators of quality. The classification considering quality characters will improve the quality and the reliability in the aircraft industry.

\subsection{Quality-Oriented Evaluation System of Civil Aircraft} Materials. AHP (Analytic Hierarchy Process) developed by Saaty is a three-layer framework of decision: objectives, criteria, and alternative solutions. It is actually a hierarchical weighted decision analysis method that is proposed by using the multiobjective comprehensive evaluation method. AHP is a widely used multicriteria decision-making tool due to its simplicity, ease of use, and great flexibility. It is particularly useful to the decision problem that the system is lacking quantitative data or is difficult to complete with quantitative analysis. It is one of the most frequently discussed multiobjective decision methods in the manufacturing industry.

The basic steps of AHP method are as follows.

(1) Construct the Hierarchy. A complex multicriteria decisionmaking problem is decomposed into a hierarchy which generally includes three layers: the top is the objective layer, the bottom is decision alternatives layer, and the middle is criteria layer.

(2) Construct Pairwise Comparison Matrix. Once the problem has been decomposed and the hierarchy is constructed, prioritization procedure starts in order to determine the relative importance of the criteria within each level. The criteria are compared pairwise according to their levels of influence and based on the specified criteria in the higher level. Then construct pairwise comparison matrix:

$$
A=\left(a_{i j}\right) n \times n=\left[\begin{array}{cccc}
a_{11} & a_{12} & \cdots & a_{1 n} \\
a_{21} & a_{22} & \cdots & a_{2 n} \\
\vdots & \vdots & \vdots & \vdots \\
a_{n 1} & a_{n 2} & \cdots & a_{n n}
\end{array}\right] .
$$

(3) Consistency Check. The quality of the output of the AHP is strictly related to the consistency of the pairwise comparison judgments. This step needs to calculate eigenvalue of maximum $\left(\lambda_{\max }\right)$ and eigenvector of the pairwise comparison matrix. Using the random consistency index (RI) and the consistency index (CI) to check the consistency,

$$
\mathrm{CR}=\frac{\mathrm{CI}}{\mathrm{RI}}, \quad \mathrm{CI}=\frac{\lambda_{\max }-n}{n-1} .
$$

Apply AHP method in aircraft material according to the above steps. Firstly, we construct the hierarchy for aircraft material. The objective layer is aircraft material classification. The quality-oriented aircraft materials evaluation system considers all the quality factors involved in airplane manufacturing process. Therefore, after considering all properties of the material in the quality management, we select "Quality basic-value," "Quality influence value," and "Quality cost" and these three properties are defined as criteria layer. We increase the subcriterion properties to quantitatively describe the detailed indicators in the quality evaluation system. Finally, we get the set for aircraft material evaluation system.

(1) The set of Quality basic-value: $A_{1}=\left(B_{11}, B_{12}, B_{13}\right)=$ (Qualification rate, Quality failure rate, and Quality stability).

(2) The set of Quality influence value: $A_{2}=\left(B_{21}, B_{22}, B_{23}\right)$ $=($ Mean Time to Repair, Impact depth, and Influence range).

(3) The set of Quality cost: $A_{3}=\left(B_{31}, B_{32}, B_{33}\right)=($ Quality management cost, Detection complexity, and Quality traceability).

The AHP model diagram is shown in Figure 1.

The illustration of each property is as follows.

(1) Quality Basic-Value. Quality basic-value directly relates to the basic quality characters of the materials. They are rigid criteria to evaluate the quality of one type of material or batches of materials. Therefore, Quality basic-value has two meanings: one is to be able to evaluate the quality of a single piece of material, which is related to the Qualification rate and failure rate of the material; the second refers to the Quality stability of a batch, commonly represented by the mean square deviation of inspection pass rate. Ultimately, we determine that the three subcriteria under Quality basicvalue include Qualification rate, Quality failure rate, and Quality stability.

(2) Quality Influence Value. Quality influence value cares more about the influence of the poor-quality materials. If 


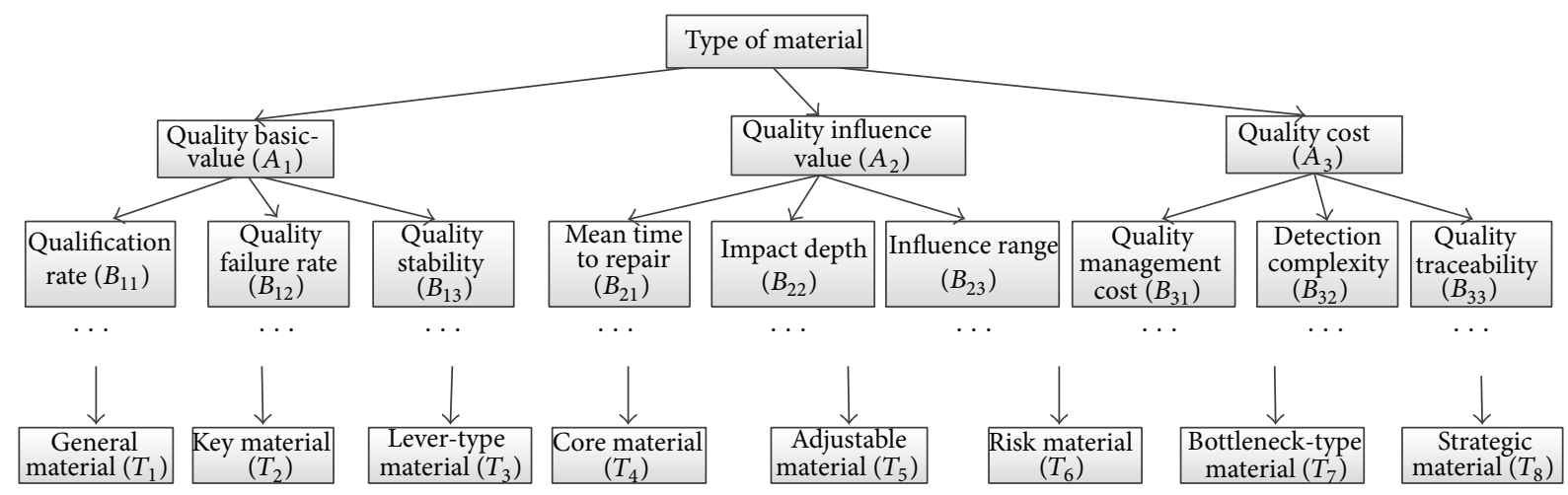

FIGURE 1: The AHP model diagram of aircraft material evaluation system.

there are poor-quality materials, we should evaluate the influence of the quality and the cost coming from the further inspection and repair. The significant materials are expected to give high priority. Finally, we determine three subcriteria under the Quality influence value which include Mean Time to Repair (MTR), Impact depth, and Influence range.

(3) Quality Cost. Quality management of civil aviation materials covers the whole life cycle. Civil aircraft production is an excelsior procedure. Most materials are required to be further improved. However, we should keep a balance. We should reduce the cost without sacrificing the quality. Therefore the attributes of the Quality cost are important. Quality cost contains Quality management cost, Detection complexity, and Quality traceability.

In this study, three criteria have the same effect proportion on the material evaluation. So, we apply hierarchy analysis, respectively, to three criteria. We get the pairwise comparison matrix $A=\left(a_{i j}\right) 3 \times 3 \quad(i, j=1,2,3)$ in which subcriteria impact on criteria layer. According to the above steps, we calculate the pairwise comparison matrix related to subcriteria $B_{11}, B_{12}, B_{13}$ and criterion $A_{1}$ :

$$
A_{1 B 1}=\left[\begin{array}{ccc}
1 & 3 & 2 \\
\frac{1}{3} & 1 & \frac{1}{2} \\
\frac{1}{2} & 2 & 1
\end{array}\right], \quad \lambda_{1 \max }=3.0092
$$

In the same way, we can also construct the pairwise comparison matrix such that $B_{21}, B_{22}, B_{23}$ impact Quality influence value $A_{2}$ and $B_{31}, B_{32}, B_{33}$ impact Quality cost $A_{3}$.

According to the following formula,

$$
w_{i}=\frac{\sum_{j=1}^{3}\left(a_{i j} / \sum_{i=1}^{3} a_{i j}\right)}{\sum_{i=1}^{3} \sum_{j=1}^{3}\left(a_{i j} / \sum_{i=1}^{3} a_{i j}\right)}, \quad i, j=1,2,3,
$$

we can calculate the weights $\left(w_{\beta}\right)$ of subcriteria to criteria layer. One has

$$
\begin{gathered}
w_{1 B 1}=[0.5390,0.1638,0.2973]^{T}, \\
w_{2 B 2}=[0.20,0.40,0.40]^{T}, \\
w_{3 B 3}=[0.5864,0.1777,0.2397]^{T} .
\end{gathered}
$$

In order to check the consistency, we use the random consistency index (RI) and the consistency index (CI) to calculate the consistency ratio (CR). The results are $\mathrm{CI}_{1}=$ $0.0079 ; \mathrm{CI}_{2}=0 ; \mathrm{CI}_{3}=0.0462$. The consistency is acceptable as long as the CR value is less than 0.1 [39]. It is obvious that the results are all less than 0.1 . That is, it successfully passes the consistency check.

Based on AHP method, we have established the material evaluation system and obtained the weights of each subcriteria impact on material evaluation.

\section{Quality-Oriented Classification System by Improved Kraljic Portfolio Matrix}

Kraljic Portfolio Matrix proposed by Peter Kraljic appeared firstly in his paper "Purchasing must become supply management." Kraljic portfolio model aims at developing differentiated purchasing and supplier strategies through classifying commodities on the basis of two dimensions: supply risk and profit impact ("low" and "high") [40]. There are four categories in the Kraljic portfolio model: leverage items, strategic items, noncritical items, and bottleneck items (Figure 2).

Quality-oriented classification system applies the thought of Kraljic Portfolio Matrix. Considering that each property on criteria layer belongs to high or low, namely, evaluation sets $V=\{$ high, low $\}$, all materials are broken down into eight categories $\left(2^{3}=8\right)$ from qualitative perspective. According to the original material classification method in accordance with material evaluation system stated above, we establish a three-dimensional model of material classification, choosing Quality basic-value as $X$ abscissa, Quality influence value as $Y$ ordinate, and Quality cost as $Z$ vertical coordinate. The threedimensional model of material classification is as shown in Figure 3. 


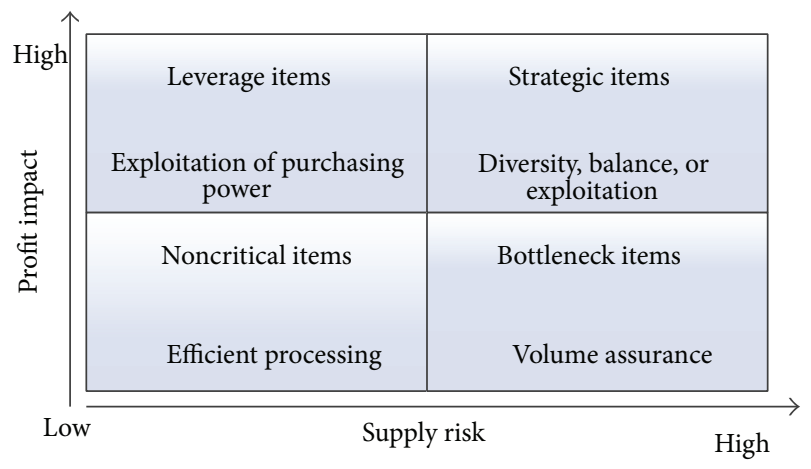

FIgure 2: Kraljic Portfolio Matrix.

As in Table 1, we make a further explanation to qualityoriented material classification in which we add a list of "Attention." With different levels of " $*$ " indicating different levels of "Attention," more "*" shows that this type of material needs more concern in the process of enterprise's quality management. Among all kinds of material, Strategic material needs the highest attention, while General material, due to its various good performances of properties, needs less attention.

(1) General Material. Standardized products generally belong to General materials. They can easily meet the quality requirements with high Quality basic-value, low Quality influence value, and low Quality cost. So, managers can pay less attention to this type of material in quality management to reduce the management cost.

(2) Key Material. Key material has high Quality basic-value and low Quality cost. However, due to the high Quality influence value, managers should also give some attention to them during the quality management.

(3) Lever-Type Material. Quality basic-value of Lever-type materials is high and their Quality influence value is low. But their expenditure of the quality management is high; managers have to consider the company's financial affordability. Therefore, quality management should optimize Quality cost. It will be fine if this type of material can be converted into General material.

(4) Core Material. The three indicated values of Core material are all very high. They have big influence on the airplane quality. Since it is difficult to manage and it is related to corporate profit, such type of material should be given high attention in quality management so that we can ensure the airplanes quality and enhance the competitiveness of enterprises.

(5) Adjustable Material. The three values of Adjustable material are all very low. On one hand this kind of material has little effect on the product and its cost is low; on the other hand, Quality basic-value of this kind of material is relatively low resulting in some potential risks. So in order to improve its Quality basic-value, we should not ignore it in the management.

(6) Risk Material. Risk material has low Quality basic-value and high Quality influence value. Such kind of materials will seriously affect the quality of the entire production once potential problems occur, not only in the depth of influence, but also in the scope. Adequate attention should be given to this type of material to minimize the risk as much as possible, and the decisions of quality management must be very prudent.

(7) Bottleneck-Type Material. The Quality basic-value of Bottleneck-type material is pretty low and its Quality influence value is not high either, but the Quality cost is very high, taking up a large proportion of the costs of operation and management. It means that we can just obtain a relatively weak quality from this type of material even though we put a large cost into them. Therefore rational planning in quality management is very necessary. Purchasing department, design department, and production department should make a good corporation to find alternatives to convert it into General material, so as to ensure the quality of the airplane.

(8) Strategic Material. Both Quality cost and Quality influence value of Strategic material are very high. Such materials have the characteristics of large quotient of fund. What is more, its quality directly influences the quality of airplane. However, due to design, technology, and other factors, it is difficult to find some other sources with high quality or irreplaceable materials. The quality problems of such materials will result in high risk. This kind of material is usually crucial to production, accounting for its large share in the final products. Besides, this situation cannot be changed in the short term even if we put a lot of money.

\section{Aircraft Material Classification Based on SVM and the Comparison with BP Neural Network}

\subsection{Introduction of Algorithm}

4.1.1. Support Vector Machine. Support Vector Machine [41] proposed by Vapnik and Cortes in 1995 is a popular small sample learning method, which has a very good performance for pattern classification problems by minimizing the Vapnik-Chervonenkis (VC) dimension and achieving a minimal structural risk. Wang et al. [42] proposed that SVM overcomes the disadvantage of big samples by obeying the rule of the minimum structural risk. It shows many unique advantages in solving small sample, nonlinear, and high-dimensional pattern recognition. Compared with other learning algorithms, SVM appears to be a good candidate for several reasons: generalization ability, without restrictive assumptions regarding the data, fast learning and evaluation for relevance feedback, and flexibility; for example, prior knowledge can be easily used to tune its kernels [43, 44]. So, it is considered as the best way for small sample classification and regression problems [45]. 


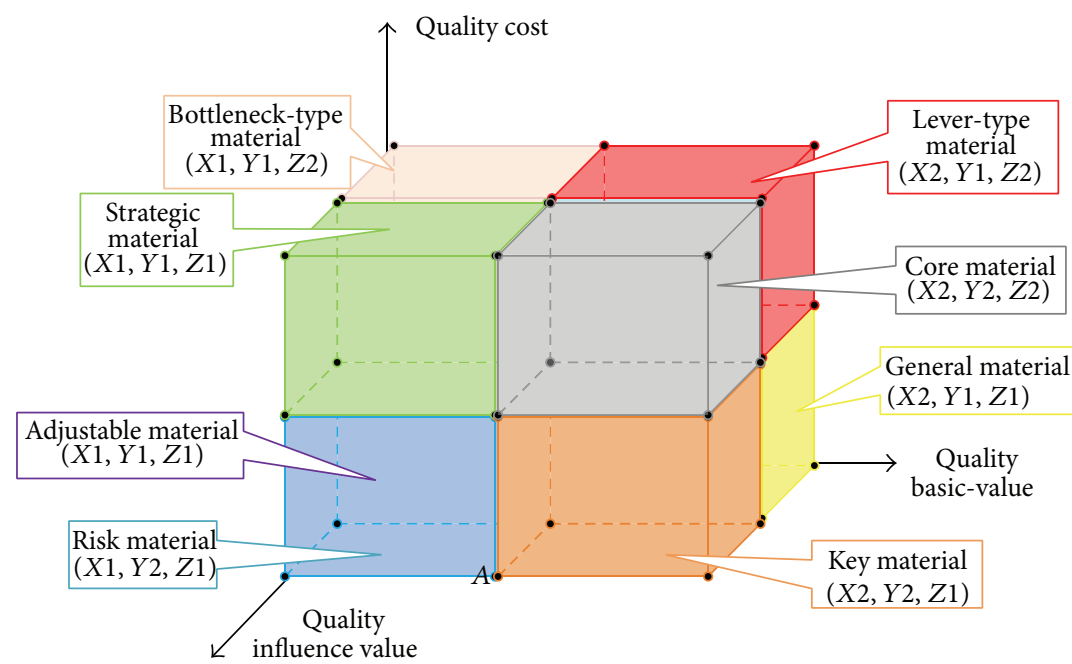

FIgURE 3: Three-dimensional model diagram of material classification.

TABLE 1: Quality-oriented material classification list.

\begin{tabular}{|c|c|c|c|c|c|}
\hline & Types & Quality eigenvalue & Quality influence value & Quality cost & Attention \\
\hline \multirow{8}{*}{$\begin{array}{l}\text { Quality-oriented material } \\
\text { classification }\end{array}$} & General material & High & Low & Low & Unnecessary \\
\hline & Key material & High & Low & High & $* *$ \\
\hline & Lever-type material & High & High & Low & $* *$ \\
\hline & Core material & High & High & High & $* * * *$ \\
\hline & Adjustable material & Low & Low & Low & $* *$ \\
\hline & Risk material & Low & Low & High & $* * * *$ \\
\hline & Bottleneck-type material & Low & High & Low & $* * *$ \\
\hline & Strategic material & Low & High & High & $* * * * *$ \\
\hline
\end{tabular}

More " $*$ " represent that we need to pay more attention to this type. For example, we should pay enough attention to strategic material with five "*" during material management, whereas we can pay less attention to key material which just has two “*”.

SVM method is evolved from optimal hyperplane of linearly separable case. In this case, the general form of hyperplane equation is $w \cdot x_{i}+b=0$. where $w$ is called hyperplane normal vector and $b$ is called hyperplane bias. The mathematical representation for classification problems is as follows:

$$
\begin{gathered}
\left.\min \phi(x)=\frac{1}{2}\|w\|^{2}, \quad \text { (namely, finding minimum }\|w\|^{2}\right), \\
y_{i}\left[w \cdot x_{i}+b\right]-1 \geq 0, \quad i=1,2, \ldots, n,
\end{gathered}
$$

However, most classification problems belong to nonlinear separable situation which need to increase the penalty parameter $C(C>0)$ and relaxation term parameter $\varepsilon_{i}\left(\varepsilon_{i} \geq\right.$ $0)$. Then the required condition of optimal hyperplane is changed into

$$
\begin{gathered}
\min \phi(x)=\frac{1}{2} \cdot\|w\|^{2}+C \cdot \sum_{i=1}^{n} \varepsilon_{i}, \\
y_{i}\left[\left(w \cdot x_{i}\right)+b\right]-1+\varepsilon_{i} \geq 0, \quad i=1,2, \ldots, n .
\end{gathered}
$$

Introducing Lagrange formula into the above equation, then the problem can be expressed as

$$
L=\frac{1}{2}\|w\|^{2}-\sum_{i} \alpha_{i}\left[y_{i}\left(w \cdot x_{i}+b\right)-1+\varepsilon_{i}\right] .
$$

Its dual problem is

$$
Q(\alpha)=\sum_{i=1}^{n} \alpha_{i}-\frac{1}{2} \sum_{i, j=1}^{n} \alpha_{i} \alpha_{j} y_{i} y_{j}\left(x_{i} x_{j}\right)
$$

When converting to a linear problem in the highdimensional space by a nonlinear exchange, the kernel function is used so that the problem can correspond to the inner product of a transform space according to relevant functional principle. At last, the corresponding classification function becomes as follows:

$$
f(x)=\operatorname{sgn}\left\{\sum_{i=1}^{n} \alpha^{*} y_{i} K\left(x_{i} \cdot x_{j}\right)+b^{*}\right\},
$$

where $\alpha^{*}$ is the optimal solution of the optimization objective function and $b^{*}$ is a classification threshold. 


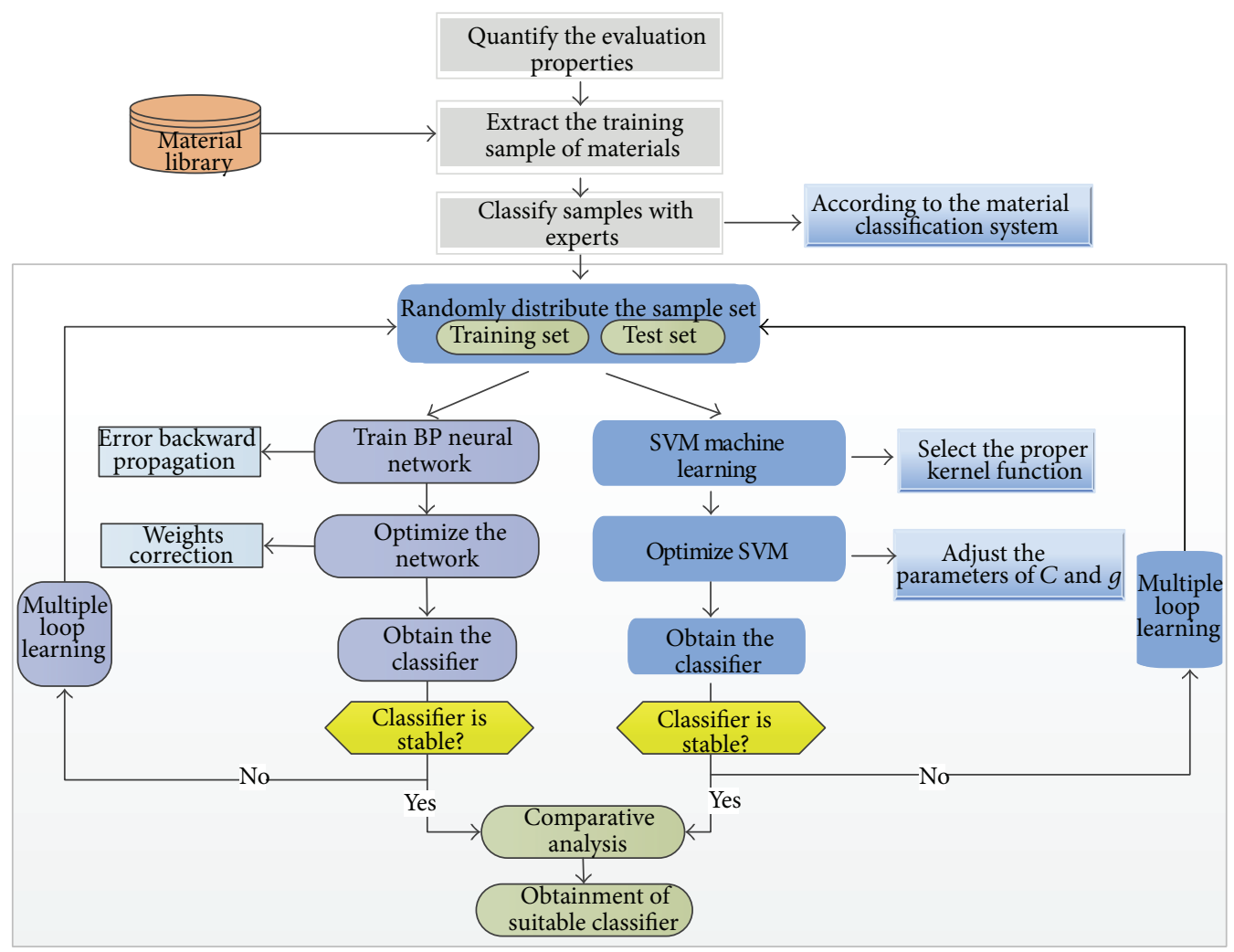

FIGURE 4: The classification process of civil aircraft materials.

4.1.2. BP Neural Network. BP neural network was put forward by the group of Rumelhart and McCelland and so forth, in 1986. As one of the widely adopted neural networks nowadays, it is a type of multilayer feed-forward network trained by an algorithm that backward propagates the errors [46]. BP network can learn and store a lot of input-output model mapping, without prior mathematical equations to reveal the mapping relationship. The topology of $\mathrm{BP}$ neural network includes input, hide, and output layer. BP neural network employs the gradient descent (GD) algorithm in learning, gradually adjusts the weights $\left(w_{i j}, T_{l i}\right)$ and thresholds $(\theta)$ of the network via the mechanism of backward propagating the errors, and eventually reaches the minimized squared error in the network.

Assuming the weight between input layer and hide layer is $w_{i j}$ and the weight between hide layer and output layer is $T_{l i}$, the calculation formulae of BP model are as follows when the desired output value is $t_{l}$.

(1) The output of hide layer is

$$
y_{i}=f\left(\sum_{j} w_{i j} x_{j}-\theta_{i}\right)=f\left(\text { net }_{i}\right) .
$$

(2) The output of output layer is

$$
\mathrm{O}_{l}=f\left(\sum_{i} w_{l i} y_{i}-\theta_{l}\right)=f\left(\text { net }_{l}\right) .
$$

$f\left(\right.$ net $\left._{l}\right)$ is output function of neuronal $S$ (Sigmoid). One has

$$
O_{l}=f\left(\text { net }_{l}\right)=\frac{1}{1+\exp \left(- \text { net }_{l}-\theta_{l}\right)} .
$$

(3) Error formula of the output layer is

$$
\begin{aligned}
E & =\frac{1}{2} \sum_{l}\left(t_{l}-O_{l}\right)^{2}=\frac{1}{2} \sum_{l}\left(t_{l}-f\left(\sum_{i} T_{l i} y_{i}-\theta_{l}\right)\right)^{2} \\
& =\frac{1}{2} \sum_{l}\left(t_{l}-f\left(\sum_{i} T_{l i} f\left(\sum_{j} w_{i j}-\theta_{i}\right)-\theta_{l}\right)\right)^{2} .
\end{aligned}
$$

In network training, the output error of output layer propagates to the hidden layer and input layer and the weight between each layer has been amended. Finally the mean square error $(E)$ of the network system gets to minimum value [47].

4.2. The Classification Study and Implementation in the Aircraft Industry. In this section, we will take one of the aircraft factory's material systems (named as Samc) as an example to illustrate our theory. The classification steps are shown in Figure 4.

(1) Quantifying the Evaluation Properties. It can easily find that some properties are nonnumeric properties in 
TABLE 2: The material quantization table.

\begin{tabular}{|c|c|c|c|c|}
\hline \multirow{2}{*}{$\begin{array}{l}\text { Criteria layer } \\
A_{1}\end{array}$} & \multirow{2}{*}{$\begin{array}{l}\text { Subcriteria layer } \\
\qquad B_{11}-B_{13}\end{array}$} & \multicolumn{3}{|c|}{ Quantitative values } \\
\hline & & 1 & 3 & 5 \\
\hline \multirow{3}{*}{ Quality basic-value } & Qualification rate & Below $90 \%$ & $90 \% \sim 95 \%$ & $95 \% \sim 100 \%$ \\
\hline & Quality failure rate & Over $10 \%$ & $3 \% \sim 10 \%$ & Below 3\% \\
\hline & Quality stability & Less stable & General & Stable \\
\hline \multirow{3}{*}{$\begin{array}{l}\text { Quality influence } \\
\text { value }\end{array}$} & Mean time to repair & Short processing time & Medium processing time & Long processing time \\
\hline & Impact depth & Weak & General & Severe \\
\hline & Influence range & Weak & General & Severe \\
\hline \multirow{3}{*}{ Quality cost } & $\begin{array}{l}\text { Quality management } \\
\text { cost }\end{array}$ & Low & Medium & High \\
\hline & Detection complexity & Simple & General & Complex \\
\hline & Quality traceability & Complete & Medium & Nonretroactivity \\
\hline
\end{tabular}

TABLE 3: The original data set.

\begin{tabular}{|c|c|c|c|c|c|c|c|c|c|}
\hline Criteria & & $A_{1}$ & & & $A_{2}$ & & & $A_{3}$ & \\
\hline Subcriteria & $B_{11}$ & $B_{12}$ & $B_{13}$ & $B_{21}$ & $B_{22}$ & $B_{23}$ & $B_{31}$ & $B_{32}$ & $B_{33}$ \\
\hline$S_{1}$ & 3 & 3 & 5 & 1 & 5 & 3 & 5 & 1 & 3 \\
\hline$S_{2}$ & 1 & 1 & 3 & 3 & 3 & 3 & 3 & 5 & 5 \\
\hline$S_{3}$ & 5 & 3 & 3 & 3 & 1 & 5 & 1 & 3 & 5 \\
\hline$S_{4}$ & 3 & 1 & 3 & 3 & 1 & 5 & 3 & 1 & 5 \\
\hline$S_{5}$ & 1 & 5 & 1 & 3 & 5 & 3 & 5 & 5 & 1 \\
\hline$S_{6}$ & 3 & 3 & 3 & 1 & 5 & 5 & 1 & 1 & 5 \\
\hline$S_{7}$ & 5 & 1 & 1 & 5 & 3 & 5 & 3 & 1 & 1 \\
\hline$S_{8}$ & 5 & 3 & 3 & 5 & 1 & 3 & 1 & 1 & 5 \\
\hline$S_{9}$ & 3 & 3 & 5 & 1 & 1 & 5 & 5 & 3 & 1 \\
\hline$S_{10}$ & 1 & 3 & 1 & 5 & 1 & 1 & 5 & 1 & 1 \\
\hline \multicolumn{10}{|l|}{$\vdots$} \\
\hline$S_{160}$ & 3 & 3 & 1 & 3 & 1 & 1 & 3 & 5 & 3 \\
\hline
\end{tabular}

the evaluation system. Firstly, we have to quantify the index. In this paper, we divide every property value into three levels: high, medium, and low, and use 5, 3, and 1 to represent them according to the property range and its numerical weight. Table 2 gives the quantitative results.

From Table 2 we can find that subcriteria directly determine the value of the three principal criteria. The weights of subcriteria were clearly appointed above in this paper. Taking radiator as an example, we symbol the Qualification rate, Quality failure rate, and Quality stability of radiator, respectively, $b_{11}, b_{12}$, and $b_{13}$. Then, Quality basic-value can be expressed as $w_{1 B 1} \times\left(b_{11}, b_{12}, b_{13}\right)$. Next we round the result: if the result is greater than or equals 3, Quality basic-value of radiator belongs to "High"; if it is less than 3, Quality basicvalue of radiator belongs to "Low." According to this method, determine the other two properties in criteria layer.

(2) Extraction of Civil Aviation Material Samples. After the quantization of aircraft material indexes, it is necessary to extract a number of samples to assess, score, and classify by the experts team. Adhering to the principles of randomness and typicality, we extract 160 materials from raw materials, system finished parts, airframe structural components, self-made parts, and accompanying equipment as sample set (Table 3).

After calculating the original data with the appointed weights, we obtained the processed data, as shown in Table 4. The last column of this table is the types of the samples based on quality-oriented classification system which we proposed in this paper. $T 1-T 8$, respectively, represents eight types of materials, namely, General material (1), Key material (2), Lever-type material (3), Core material (4), Adjustable material (5), Risk material (6), Bottleneck-type material (7), and Strategic material (8).

(3) Establishment of Classifier. This study selects MATLAB as the testing platform to verify the result and evaluate the differences of SVM and BP neural network. Selecting appropriate kernel function type and kernel parameters proves crucial [48], because it determines the performance of the learning and generalization ability. Since the RBF kernel function has good performance and it shows good efficiency in practical problems, this study adopts RBF kernel. In practical applications, in order to reduce dependence on the initial sample and improve the accuracy of SVM classifier, it is important to optimize the parameters of $C$ (penalty 
TABLE 4: The processed data.

\begin{tabular}{lcccc}
\hline & $A_{1}$ & $A_{2}$ & $A_{3}$ & $\begin{array}{c}\text { The types of } \\
\text { material }\end{array}$ \\
\hline$S_{1}$ & 3.5949 & 3.4 & 3.8288 & 4 \\
$S_{2}$ & 1.5947 & 3 & 3.8462 & 8 \\
$S_{3}$ & 4.0783 & 3 & 2.318 & 3 \\
$S_{4}$ & 2.6727 & 3 & 3.1354 & 8 \\
$S_{5}$ & 1.6553 & 3.8 & 4.0602 & 8 \\
$S_{6}$ & 3.0003 & 4.2 & 1.9626 & 3 \\
$S_{7}$ & 3.1561 & 4.2 & 2.1766 & 3 \\
$S_{8}$ & 4.0783 & 2.6 & 1.9626 & 1 \\
$S_{9}$ & 3.5949 & 2.6 & 3.7048 & 2 \\
$S_{10}$ & 1.3277 & 1.8 & 3.3494 & 6 \\
$\vdots$ & & & & \\
$S_{160}$ & 2.4057 & 1.4 & 3.3668 & 6 \\
\hline
\end{tabular}

factor) and $g$ (variance of RBF kernel function). This study chooses to use cross-validation method to find the optimal parameters of $C$ and $g$.

During the experiment of BP neural network, its training step is 1000 , error goal is 0.01 , the maximum convergence time is 1000 , and training and learning rate is 0.1 .

Without loss of generality, randomly select 130 samples as training samples from the 160 proceeded data. The remaining 30 samples are set as test samples. Because the training set and test set are randomly generated, the running results will be different each time. We randomly conducted a simulation test, the accuracy rate of training samples by SVM was $100 \%$, and the accuracy rate of test samples was $96.6667 \%$. However, prediction result of BP neural network is not very desirable. Its prediction accuracy is pretty low. Additionally, the results belong to two types sometimes, leading to the fact that we need to judge forecast situation by ourselves. The results of the simulation test are shown in Figures 5 and 6.

It is obvious that the classification accuracy of SVM classifier is better than BP neural network classifier. In other words, the learning rate and efficiency of the former is much better than the latter. We also found that, in the experimental process, the accurate rate of SVM classifier was above $95 \%$ through many experiments. And multiple learning can also enhance the accuracy of BP neural network. When BP neural network classifier was stable, take 10 times cyclic forecast of sample set. The predicted results showed that the average accuracy rate was only $84.333 \%$. What is worse, the tenth prediction accuracy was less than $60 \%$. Compared with BP neural network, higher classification accuracy can be obtained by SVM without a lot of training. Similarly, randomly take 10 times forecast. The average accuracy rate was $96 \%$, far better than BP neural network, and extreme minimum condition did not appear. That is, the classification result of SVM is ideal which can be used to classify aircraft materials.

From Table 5 we can clearly find that SVM algorithm has great advantages in small sample classification, and it is suitable for civil aircraft materials classification.

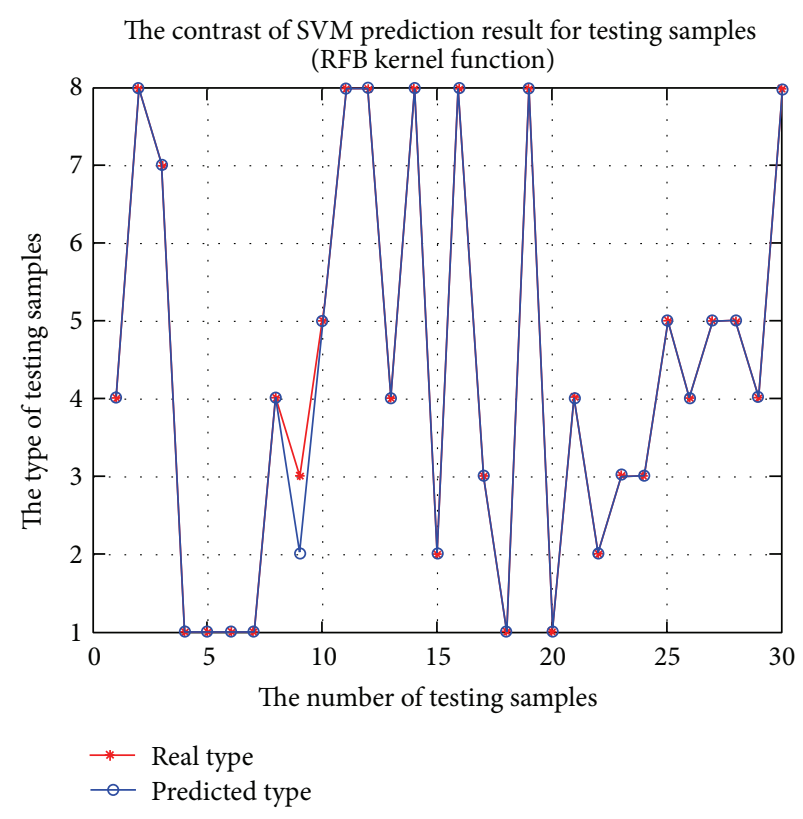

FIGURE 5: Prediction result of test samples by SVM.

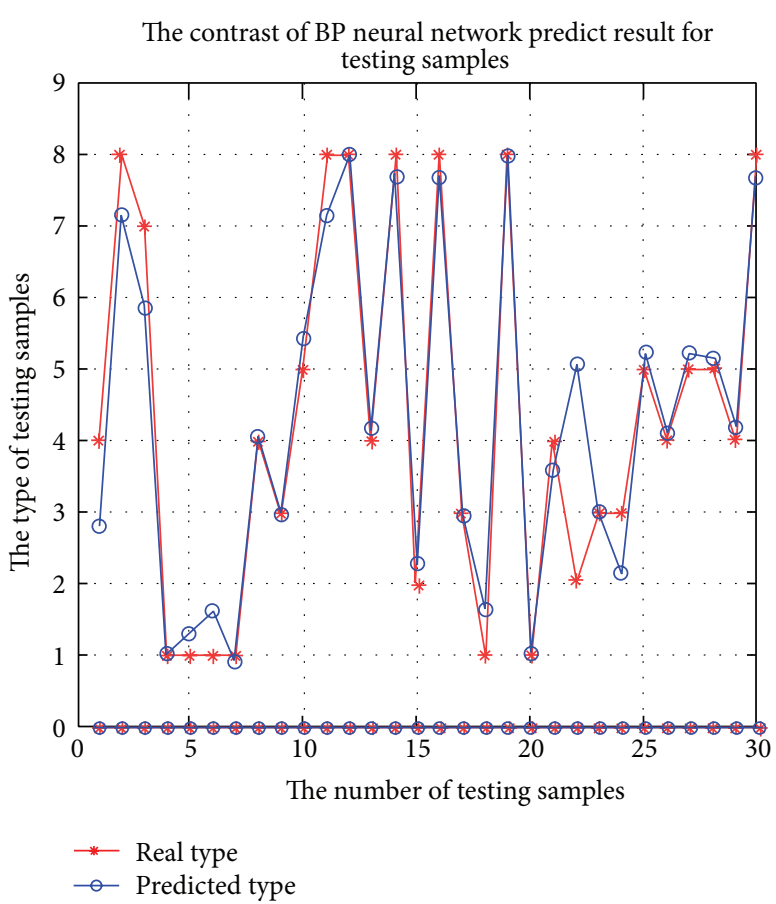

FIGURE 6: Prediction result of test samples by BP neural network.

\section{Conclusion}

We propose a quality-oriented material classification considering the quality character, Quality influence, and Quality cost. The quality-oriented material evaluation system is established to quantify each property by AHP method. Through improving conventional Kraljic Portfolio Matrix, we establish a three-dimensional material classification model, which divides the aircraft materials into eight categories: General 
TABLE 5: Comparison of the prediction results of BP neural network and SVM.

\begin{tabular}{lcc}
\hline Sequence number & \multicolumn{2}{c}{ Accuracy } \\
\hline 1 & BP neural network & SVM \\
2 & $73.333 \%$ & $100.0 \%$ \\
3 & $86.667 \%$ & $96.667 \%$ \\
4 & $83.333 \%$ & $100.0 \%$ \\
5 & $86.667 \%$ & $100.0 \%$ \\
6 & $86.667 \%$ & $90.0 \%$ \\
7 & $93.333 \%$ & $86.667 \%$ \\
8 & $96.667 \%$ & $100.0 \%$ \\
9 & $93.333 \%$ & $100.0 \%$ \\
10 & $86.667 \%$ & $96.667 \%$ \\
Average & $56.667 \%$ & $90.0 \%$ \\
\hline
\end{tabular}

material, Key material, and so on. Compared with the BP neural network, the study verifies that SVM algorithm has a good advantage in the classification of multiple small samples in the aircraft industry. The contribution of this paper lies in two aspects: in general, the material classification is oriented to inventory management based on $\mathrm{ABC}$ inventory control and so on. However, different materials have different qualityrelated attributes, especially in the aircraft industry. After fully evaluating the quality-related attributes in the aircraft industry, we apply AHP and improve conventional Kraljic Portfolio Matrix to establish a three-dimensional material classification model. Therefore, we could take different strategies for the different materials to improve the quality and reduce the cost in the quality management of the aircraft industry. On the other hand, this study not only provides a new idea for manufacturing material classification to improve the quality management, but also proves it is feasible to apply SVM in civil aviation material classification. We all know that SVM shows many unique advantages in solving small sample, nonlinear, and high-dimensional pattern recognition. Although the SVM is applied extensively as the best way for small sample classification and regression problems, it has not been used in the aircraft material classification. There are a large number of component parts of an aircraft, more than millions. However, each part is few. The case study shows the SVM is effective to solve the classification when the categories are extensive and the sample is very small. However, we should do further research. For example, taking into account the practicability, we now only consider the level of low and high in the evaluating indicator. If possible, we should try to supplement level of "medium," and the classified management for the quality may be more considerate. Although we have verified this classification model in an aircraft company, it is not enough. We should try to improve this model in this company and the other aircraft companies, and even the other industries. We should further propose the specific management strategies to the different category of material and evaluate the effectiveness, so that we could know the value of this research indeed.

\section{Conflict of Interests}

The authors declare that there is no conflict of interests regarding the publication of this paper.

\section{Acknowledgments}

This work is supported by Shanghai Science and Technology Committee under Grants nos. 12dz1124300 and 13521103604. It is also supported by Shanghai Key Laboratory of Intelligent Manufacturing and Robotics. The authors are grateful for the financial support and also would like to thank the anonymous reviewers and the editor for their comments and suggestions.

\section{References}

[1] T. D’Orazio, C. Guaragnella, M. Leo, and P. Spagnolo, "Defect detection in aircraft composites by using a neural approach in the analysis of thermographic images," NDT and E International, vol. 38, no. 8, pp. 665-673, 2005.

[2] H. F. Dickie, "ABC inventory analysis shoots for dollars not pennies," Factory Management and Maintenance, vol. 109, pp. 92-94, 1951.

[3] Z. Zhao, K. Ji, X. Xing, W. Chen, and H. Zou, "Ship classification with high resolution terrasar-x imagery based on analytic hierarchy process," International Journal of Antennas and Propagation, vol. 2013, Article ID 698370, 13 pages, 2013.

[4] T. L. Saaty, What is the Analytic Hierarchy Process? Springer, Berlin, Germany, 1988.

[5] J. Moran, S. Marsh, S. Nakui, and G. Hoffherr, "Design concept evaluation in product development using rough sets and grey relation analysis," Journal of Mechanical Design, vol. 124, no. 3, pp. 385-392, 1991.

[6] W. Pedrycz and M. Song, "Analytic Hierarchy process (AHP) in group decision making and its optimization with an allocation of information granularity," IEEE Transactions on Fuzzy Systems, vol. 19, no. 3, pp. 527-539, 2011.

[7] C. K. Kwong and H. Bai, "A fuzzy AHP approach to the determination of importance weights of customer requirements in quality function deployment," Journal of Intelligent Manufacturing, vol. 13, no. 5, pp. 367-377, 2002.

[8] D. M. Lee and P. R. Drake, "A portfolio model for component purchasing strategy and the case study of two South Korean elevator manufacturers," International Journal of Production Research, vol. 48, no. 22, pp. 6651-6682, 2010.

[9] R. Terpend, D. R. Krause, and K. J. Dooley, "Managing buyersupplier relationships: empirical patterns of strategy formulation in industrial purchasing," Journal of Supply Chain Management, vol. 47, no. 1, pp. 73-94, 2011.

[10] H. A. Guvenir and E. Erel, "Multicriteria inventory classification using a genetic algorithm," European Journal of Operational Research, vol. 105, no. 1, pp. 29-37, 1998.

[11] F. Y. Partovi and M. Anandarajan, "Classifying inventory using an artificial neural network approach," Computers and Industrial Engineering, vol. 41, no. 4, pp. 389-404, 2001.

[12] W. E. Henley and D. J. Hand, "A k-nearest-neighbour classifier for assessing consumer credit risk," The Statistician, vol. 45, no. 1, pp. 77-95, 1996. 
[13] C.-Y. Tsai and S.-W. Yeh, "A multiple objective particle swarm optimization approach for inventory classification," International Journal of Production Economics, vol. 114, no. 2, pp. 656666, 2008.

[14] H. Yin, S. Yang, X. Zhu, S. Jin, and X. Wang, "Satellite fault diagnosis using support vector machines based on a hybrid voting mechanism," The Scientific World Journal, vol. 2014, Article ID 582042, 11 pages, 2014.

[15] C. Fernandez-Lozano, C. Canto, M. Gestal et al., "Hybrid model based on genetic algorithms and SVM applied to variable selection within fruit juice classification," The Scientific World Journal, vol. 2013, Article ID 982438, 13 pages, 2013.

[16] L. Gao, B.-S. Tong, and X.-H. Dong, "Research on component and supplier management in collaborative design," Computer Integrated Manufacturing Systems, vol. 8, no. 10, pp. 766-769, 2002.

[17] S. B. Kotsiantis, "Supervised machine learning: a review of classification techniques," Informatica, vol. 31, no. 3, pp. 249268, 2007.

[18] T. Hastie, R. Tibshirani, and J. Friedman, The Elements of Statistical Learning, vol. 2, Springer, New York, NY, USA, 2009.

[19] Z. Zuo, Y. Hu, Q. Li, and L. Zhang, "Data mining of the thermal performance of cool-pipes in massive concrete via in situ monitoring," Mathematical Problems in Engineering, vol. 2014, Article ID 985659, 15 pages, 2014.

[20] G. Zhu and D. G. Blumberg, "Classification using ASTER data and SVM algorithms: the case study of Beer Sheva, Israel," Remote Sensing of Environment, vol. 80, no. 2, pp. 233-240, 2002.

[21] R. Venegas, "Academic text classification based on lexicalsemantic content," 2014.

[22] L. P. Hernández, J. M. Flórez, and J. B. Cebayos, "A linear approach to determining an SVM-based fault locator's optimal parameters," Ingenieria e Investigacion, vol. 29, no. 1, pp. 76-81, 2009.

[23] J. W.-H. Kao, S. M. Berber, and V. Kecman, "Blind multiuser detector for chaos-based CDMA using support vector machine," IEEE Transactions on Neural Networks, vol. 21, no. 8, pp. 1221-1231, 2010.

[24] X. Yu, F. Chu, and R. Hao, "Fault diagnosis approach for rolling bearing based on support vector machine and soft morphological filters," Journal of Mechanical Engineering, vol. 45, no. 7, pp. 75-80, 2009.

[25] C. Wei, "Real-time vehicle classification with GA, PCA and improved SVM," Journal of Eastern Liaoning University (Natural Science), vol. 17, no. 4, pp. 296-302, 2012.

[26] X.-X. Ma, X.-Y. Huang, and Y. Chai, "PTMC classification algorithm based on support vector machines and its application to fault diagnosis," Control and Decision, vol. 18, no. 3, pp. 272284, 2003.

[27] Z. Yixin, Design and Implementation of the Integrated Material Management System for ARJ21, Fudan University, Shanghai, China, 2011.

[28] A. A. Ghobbar and C. H. Friend, "Evaluation of forecasting methods for intermittent parts demand in the field of aviation: a predictive model," Computers and Operations Research, vol. 30, no. 14, pp. 2097-2114, 2003.

[29] C. H. Friend, A. Louise Swift, and A. A. Ghobbar, "A predictive cost model in lot-sizing methodology, with specific reference to aircraft parts inventory: an appraisal," Production and Inventory Management Journal, vol. 42, no. 3-4, pp. 24-33, 2001.
[30] Y. Xu, Research on Key Techniques of Spares Data Management Based on PDM, Nanjing University of Aeronautics and Astronautics, Nanjing, China, 2008.

[31] L. Sun and H. Zuo, "Multi-echelon inventory optimal model of civil aircraft spare parts," in Proceedings of the Chinese Control and Decision Conference (CCDC '10), pp. 824-828, May 2010.

[32] G. Ning, Research on Primary Inventory of Civil Aircraft Spare Parts for Manufacturer, Nanjing University of Aeronautics and Astronautics, Nanjing, China, 2008.

[33] H. X. Cai, M. Y. Dai, and T. Yu, "Material coding for aircraft manufacturing industry," Journal of Aerospace Technology and Management, vol. 6, no. 2, pp. 183-191, 2014.

[34] W.-W. Wu, H.-X. Cai, L. Wang, F. Xiong, and T. Yu, "Research on inventory control based on material segmentation of civil aviation," Informatization of Chinese Manufacturing Industry, vol. 39, no. 1, pp. 13-18, 2010.

[35] V. Mancini, M. Pasquali, and M. M. Schiraldi, "Opportunities for using RFID in the aircraft production process," International Journal of RF Technologies: Research and Applications, vol. 3, no. 4, pp. 243-255, 2012.

[36] D. P. Davies, S. L. Jenkins, and F. R. Belben, "Survey of fatigue failures in helicopter components and some lessons learnt," Engineering Failure Analysis, vol. 32, pp. 134-151, 2013.

[37] R. E. Shalin and I. A. Kantsevich, "Quality of aircraft materials as a guarantee of aircraft quality and reliability," Elektrokhimiya, vol. 32 , no. 62 , pp. 75-76, 1996.

[38] R. W. Hillermeier, K. Chung, J. C. Seferis, and M. H. Diaz, "Reduced quality assurance testing of commercial aircraft prepreg systems," in Proceedings of the 45th International SAMPE Symposium and Exhibition, May 2000.

[39] E. Kiliç Delice and Z. Güngör, "The usability analysis with heuristic evaluation and analytic hierarchy process," International Journal of Industrial Ergonomics, vol. 39, no. 6, pp. 934939, 2009.

[40] S. S. Padhi, S. M. Wagner, and V. Aggarwal, "Positioning of commodities using the Kraljic Portfolio Matrix," Journal of Purchasing and Supply Management, vol. 18, no. 1, pp. 1-8, 2012.

[41] V. N. Vapnik, The Nature of Statistical Learning Theory, Statistics for Engineering and Information Science, Springer, New York, NY, USA, 2nd edition, 2000.

[42] M. Wang, P. Wang, J.-S. Lin, X. Li, and X. Qin, "Nonlinear inertia classification model and application," Mathematical Problems in Engineering, vol. 2014, Article ID 987686, 9 pages, 2014.

[43] C. Zhang, X. Chen, M. Chen, S.-C. Chen, and M.-L. Shyu, "A multiple instance learning approach for content based image retrieval using one-class support vector machine," in Proceedings of the IEEE International Conference on Multimedia and Expo (ICME '05), pp. 1142-1145, IEEE, July 2005.

[44] L. Zhang, F. Lin, and B. Zhang, "Support vector machine learning for image retrieval," in Proceedings of the IEEE International Conference on Image Processing (ICIP '01), vol. 2, pp. 721-724, October 2001.

[45] L. Zheng, H. Zhou, C. Wang, and K. Cen, "Combining support vector regression and ant colony optimization to reduce NOx emissions in coal-fired utility boilers," Energy and Fuels, vol. 22, no. 2, pp. 1034-1040, 2008.

[46] L. Zhuo, J. Zhang, P. Dong, Y. Zhao, and B. Peng, "An SA-GABP neural network-based color correction algorithm for TCM tongue images," Neurocomputing, vol. 134, pp. 111-116, 2014. 
[47] Y. Zhu, W. Zeng, Y. Sun, F. Feng, and Y. Zhou, "Artificial neural network approach to predict the flow stress in the isothermal compression of as-cast TC21 titanium alloy," Computational Materials Science, vol. 50, no. 5, pp. 1785-1790, 2011.

[48] B. Caputo, E. Hayman, and P. Mallikarjuna, "Class-specific material categorisation," in Proceedings 10th IEEE International Conference on Computer Vision (ICCV '05), vol. 2, pp. 15971604, IEEE, October 2005. 


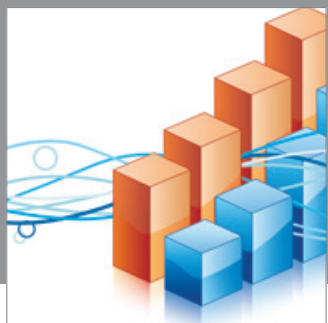

Advances in

Operations Research

mansans

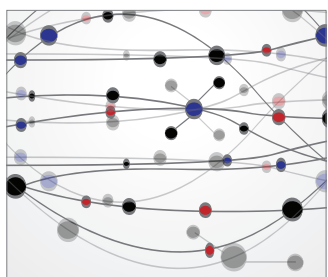

The Scientific World Journal
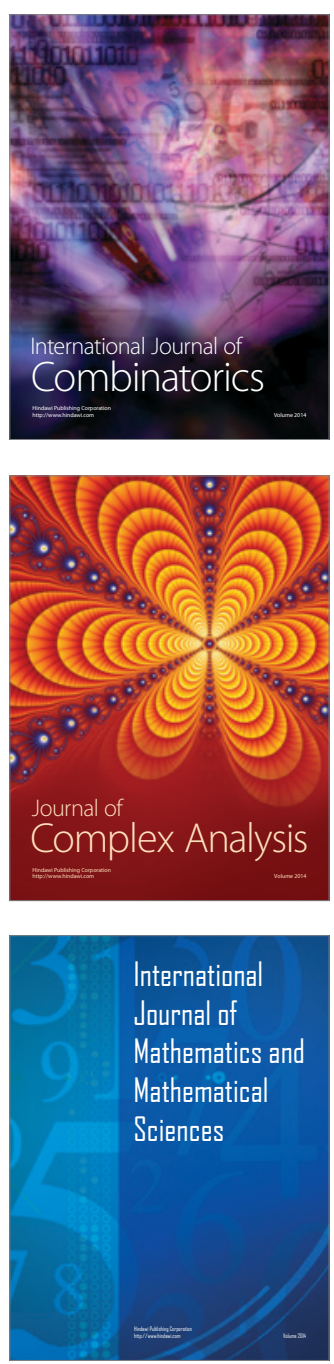
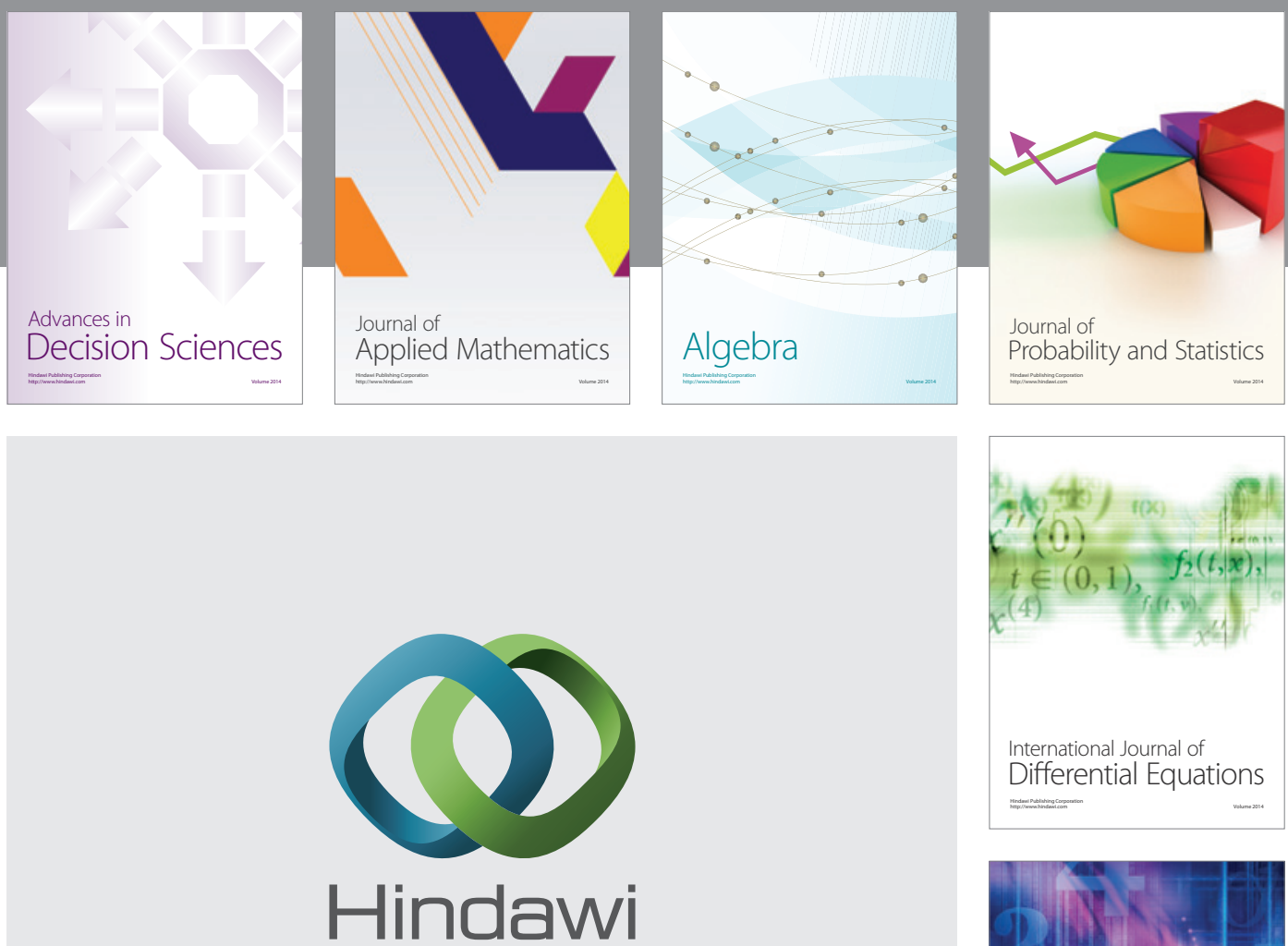

Submit your manuscripts at http://www.hindawi.com
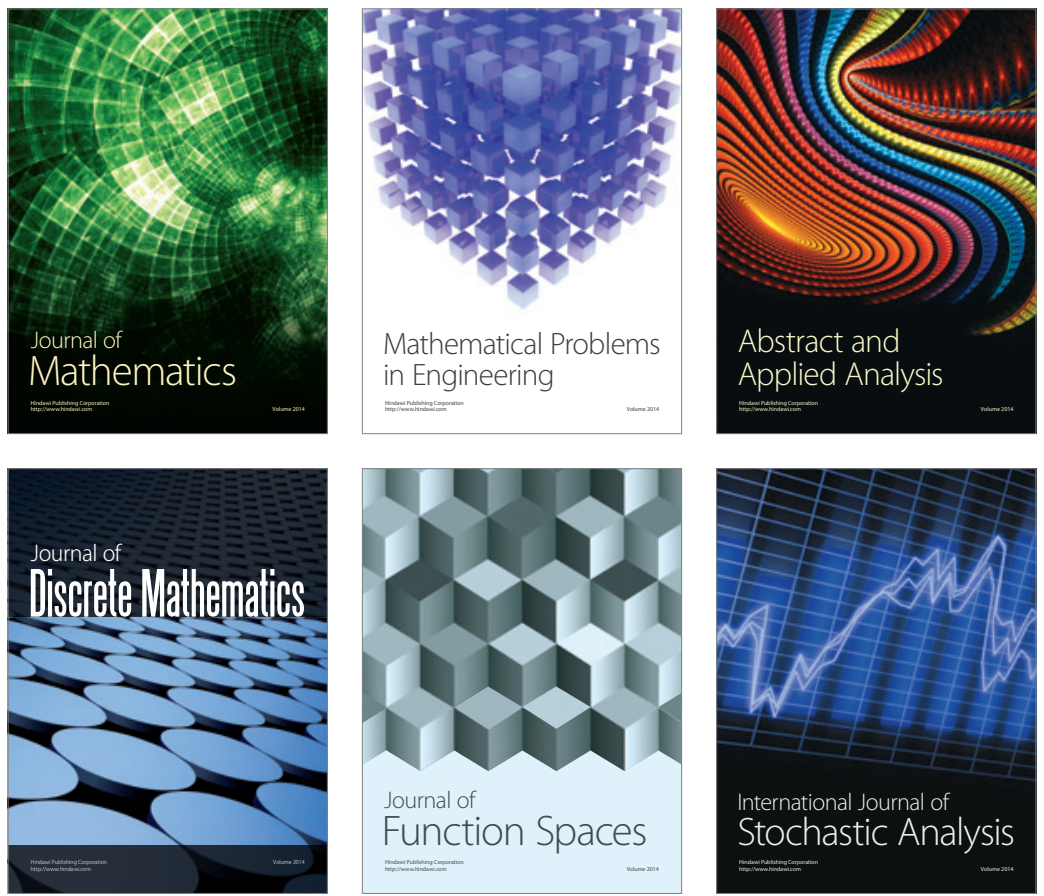

Journal of

Function Spaces

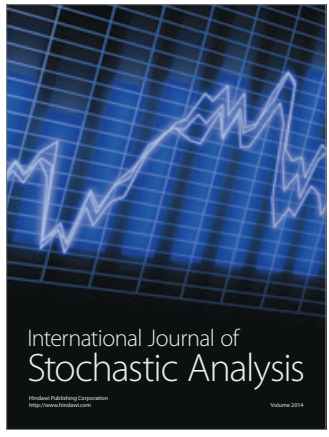

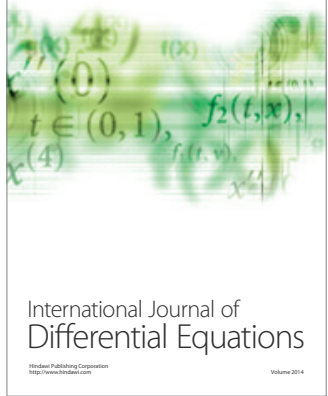
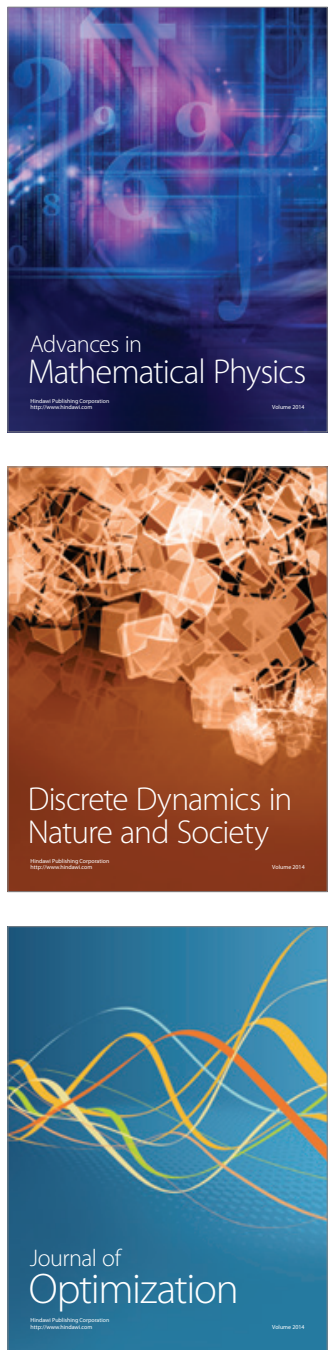